\title{
Workflow Automation for a Virtual Hypertension Management Program
}

\author{
William J. Gordon ${ }^{1,2,3}$ Alexander J. Blood ${ }^{1}$ Kira Chaney $^{3}$ Eugene Clark $^{3}$ Corey Glynn ${ }^{3}$
}

Remlee Green ${ }^{3}$ John St. Laurent ${ }^{3}$ Charlotte Mailly ${ }^{3}$ Marian McPartlin ${ }^{3}$ Shawn Murphy ${ }^{2,3,4}$

Hunter Nichols ${ }^{3}$ Michael Oates ${ }^{3}$ Samantha Subramaniam ${ }^{3}$ Matthew Varugheese ${ }^{3}$

Kavishwar Wagholikar ${ }^{2,4}$ Samuel Aronson*3 Benjamin M. Scirica*1,2,3

\footnotetext{
${ }^{1}$ Department of Medicine, Brigham and Women's Hospital, Boston, Massachusetts, United States

2 Harvard Medical School, Boston, Massachusetts, United States

3 Mass General Brigham, Boston, Massachusetts, United States

${ }^{4}$ Department of Medicine, Massachusetts General Hospital, Boston, Massachusetts, United States
}

Appl Clin Inform 2021;12:1041-1048.
Address for correspondence William J. Gordon, MD, MBI, BWH Hospitalist Service, PBB-B4-428, 75 Francis Street, Boston, MA 02115, United States (e-mail: wjgordon@partners.org).

\section{Abstract}

Keywords

- workflow automation

- clinical innovation

- blood pressure monitoring

- customer relationship management
Objectives Hypertension is a modifiable risk factor for numerous comorbidities and treating hypertension can greatly improve health outcomes. We sought to increase the efficiency of a virtual hypertension management program through workflow automation processes.

Methods We developed a customer relationship management (CRM) solution at our institution for the purpose of improving processes and workflow for a virtual hypertension management program and describe here the development, implementation, and initial experience of this CRM system.

Results Notable system features include task automation, patient data capture, multichannel communication, integration with our electronic health record (EHR), and device integration (for blood pressure cuffs). In the five stages of our program (intake and eligibility screening, enrollment, device configuration/setup, medication titration, and maintenance), we describe some of the key process improvements and workflow automations that are enabled using our CRM platform, like automatic reminders to capture blood pressure data and present these data to our clinical team when ready for clinical decision making. We also describe key limitations of CRM, like balancing out-of-the-box functionality with development flexibility. Among our first group of referred patients, 76\% (39/51) preferred email as their communication method, 26/51 (51\%) were able to enroll electronically, and $63 \%$ of those enrolled (32/51) were able to transmit blood pressure data without phone support.

Conclusion A CRM platform could improve clinical processes through multiple pathways, including workflow automation, multi-channel communication, and device integration. Future work will examine the operational improvements of this health information technology solution as well as assess clinical outcomes.

* These authors contributed equally to this manuscript.

received

June 3, 2021

accepted after revision

September 23, 2021
DOI https://doi.org/

10.1055/s-0041-1739195

ISSN 1869-0327. (c) 2021. The Author(s).

This is an open access article published by Thieme under the terms of the Creative Commons Attribution-NonDerivative-NonCommercial-License, permitting copying and reproduction so long as the original work is given appropriate credit. Contents may not be used for commercial purposes, or adapted, remixed, transformed or built upon. (https://creativecommons.org/ licenses/by-nc-nd/4.0/)

Georg Thieme Verlag KG, Rüdigerstraße 14, 70469 Stuttgart,

Germany 


\section{Background and Significance}

Hypertension remains an important modifiable risk factor for cerebrovascular and cardiovascular disease, and treating hypertension leads to improved morbidity and mortality. ${ }^{1-3}$ Despite this, an unacceptable number of patients are not adequately treated for hypertension, even in the presence of generic medications and robust, international guidelines for management. ${ }^{4}$ Recent evidence suggests that improvements in hypertension control and associated mortality achieved in the early 2000s have subsequently worsened. ${ }^{5}$ The World Health Organization estimates that more than 1 billion people have hypertension worldwide, but less than one in five are well controlled. ${ }^{6}$ Novel methods of improving hypertension management would have substantial public health benefits.

Prior work has shown that a remote, algorithmically driven, navigator and pharmacist-led hypertension program could improve blood pressure control in a diverse population of patients. ${ }^{7}$ This clinical program, developed and run at Mass General Brigham, a non-profit health care system in Boston, Massachusetts, relies on non-clinical staff ("navigators") to virtually manage patients referred to us directly through providers or through population-health outreach efforts. Patients are managed remotely, and besides having laboratories checked, do not have any in-person interactions with our program. Navigators are supported through clinical pharmacists, operating under a collaborative practice agreement, ${ }^{8}$ with physicians supervising the pharmacists. The navigators and pharmacists are further supported by guideline-based algorithms that guide medication titration decisions, along with other clinical requirements, like timing of laboratory follow-up and scheduled disease-state evaluations. This program, started in 2017, has been particularly valuable during the COVID pandemic, and has enabled chronic disease management without the risks of in-person care. $^{9-11}$

The clinical program has had strong outcomes including significant, sustained, and rapid reductions in systolic and diastolic blood pressures and we were interested in scaling the program to more patients, as the existing program used several legacy systems to drive management. In an attempt to do this, we built and piloted a customer relationship management (CRM) software product. CRM systems are important enterprise technology systems that allow businesses to manage customer relationships through workflow, data capture, and other processes. ${ }^{12}$ For example, a store may use a CRM system to keep a record of customer purchasing habits. This store could then directly market to a customer when a new product becomes available that aligns with their prior purchases. Used strategically, CRM systems can span an entire organization and serve as the backbone for all customer relationships. CRM software is a major industryworldwide spending on CRM software is estimated to be more than $\$ 48$ billion dollars. ${ }^{13}$

In the health care context, a CRM system allows a provider organization to build stronger patient relationships. The CRM can track patient medical and demographic character- istics, patient preferences, and interactions the patient has had with the health care system, and health care CRM implementations are beginning to emerge. One example is using CRM to track the activity and program participation levels of the residents of a nursing home, to look for changes that may indicate a clinical decline, as well as tracking resident's family members and their feedback. ${ }^{14}$ Care processes can be built on top of CRM, for example, scheduling follow-up visits, ensuring patients are up-to-date on recommended screening, or engaging patients so that they can complete tasks critical to optimizing their health including obtaining laboratories, imaging studies, or taking blood pressure measurements. ${ }^{15}$ Importantly, CRM enables significant workflow automation. Processes that previously required manual intervention or tracking can be automatically identified and subsequently managed, from simple reminders to complex alerts built around clinical events, laboratory, or imaging results. These process automations could dramatically improve care-for example, ensuring follow-up of all abnormal results, improving employee efficiency, or reducing unnecessary manual tasks. However, automation also has drawbacks-less human interaction, loss of flexibility, and new errors or hazards created through automations, like alert fatigue.

\section{Objectives}

Because of these benefits, we developed a CRM solution at our institution for the purpose of improving processes and workflow for a virtual hypertension management program. A principal goal of the initiative was scaling our program to substantially more patients, which we hoped a CRM solution would enable. We describe the development and implementation of this CRM system, including selected system features, a detailed description of some of the workflow processes we have automated because of this CRM implementation, and results from our first tranche of referrals which served as an initial pilot before scaling more broadly.

\section{Methods}

\section{System Description}

We built our CRM system using Microsoft Dynamics 365 (D365), a CRM solution and component of the Microsoft PowerPlatform. D365 enables "low-code" development, ${ }^{16}$ with the flexibility to add custom-coded components as needed. D365 and the PowerPlatform are cloud-based (Azure) Software as a Service (SaaS) offerings and provide a HIPAAcompliant application development environment. We chose to develop in this environment due to the out-of-the-box capabilities that would accelerate our development timelines, as well as potential to improve workflow automation and other efficiencies as outlined below. In addition to patient management and workflow tools, our system includes a rule-based decision support aid for guiding management decisions and read-based integrations from our EHR (electronic health record) (Epic Systems, Verona, Wisconsin, United States), for example, laboratory data and patient demographics. 
Additional modules used include a survey tool to collect patient-reported data and the D365 Field Service module. Customization of our CRM system generally fell into two categories. First was functionality that required engineering resources and programming. For example, our CRM system did not have native EHR integration capabilities, so we wrote custom software code to enable those connections. The second category of functionality did not require programming and could generally be described as configuration. Examples include adding a new field to an existing form or generating a new view of a CRM data element.

Blood pressure data are collected from cellularly-enabled devices (BlipCare, Inc.) mailed to patients at enrollment. Blood pressure data are directly integrated into the CRM system by exposing an application programming interface which allows the device vendor to transmit blood pressure data into our system in real-time, mapped to patients by device ID (set during patient enrollment). The system is deployed at Mass General Brigham (Boston, Massachusetts, United States).

\section{System Design Process}

We convened a multidisciplinary working group at the beginning of development to ensure all stakeholders were represented. Participants included our technical team, product team, design team, clinical navigators, pharmacists, physicians, and clinical operations leads. We held several design sessions where we walked through current workflows to look for opportunities for improvement. Using principles of agile methodology, we created a "minimum viable product" that allowed us to launch an initial version of the CRM system. Initially, the CRM system required significant manual processes, but we continued to build functionality to decrease manual work as we learned what was needed and worked best from real clinical examples. We held daily "stand ups" with our clinical and technical teams where we observed our navigators managing patients, which facilitated rapid iteration and development of new tools.

\section{Results}

Our CRM system has several notable features, highlighted in - Table 1, which we hope will lead to improved processes, workflow automation, and high-quality care delivery. First, the system deploys task automation processes. Tasks are often created automatically, assigned to individuals or teams, and given due dates. For example, a new patient referral triggers a task to "screen" the patient for program eligibility. All enrolled patients in our program must always have a task or active workflow assigned to them, and the system checks nightly to ensure that there is a workflow or task assigned to every patient and alerts the navigator if these conditions are not met. Second, we have built-in survey capabilities that allow us to seamlessly collect patient-reported outcomes or other patient-generated information, with responses integrated directly into the system. Third, we have built multichannel communication capabilities. Emails and short message service (SMS) can be sent directly from the system, while other communication methods-like phone, or patient portal messages-can be tracked through task creation and completion. Finally, we have built integrations from our EHR, such as using FHIR services for laboratory results. Patient

Table 1 Selected system features of our CRM deployment

\begin{tabular}{|l|l|l|}
\hline System feature & Description & Example \\
\hline Task automation & $\begin{array}{l}\text { The CRM system provides task } \\
\text { generation, assignment, and } \\
\text { automation to guide workflows, } \\
\text { and ensure appropriate follow-up }\end{array}$ & $\begin{array}{l}\text { - When a blood pressure cuff is sent to a patient, a } \\
\text { reminder will automatically trigger a navigator phone } \\
\text { call in } 7 \text { days, unless the patient begins checking their } \\
\text { blood pressure } \\
\text { The system checks daily to make sure every patient has } \\
\text { either a task or workflow assigned, ensuring no patients } \\
\text { are "lost" }\end{array}$ \\
\hline Patient data capture & $\begin{array}{l}\text { Electronic survey tools to capture } \\
\text { key information from patients, like } \\
\text { current medications and pregnancy } \\
\text { status. }\end{array}$ & $\begin{array}{l}\text { - Survey sent to referred patients to determine } \\
\text { communication preferences }\end{array}$ \\
\hline $\begin{array}{l}\text { Multi-channel } \\
\text { communication }\end{array}$ & $\begin{array}{l}\text { Ability to email or SMS patients } \\
\text { directly through the software, to } \\
\text { improve access, decrease phone } \\
\text { calls, and reduce friction in } \\
\text { communication. }\end{array}$ & $\begin{array}{l}\text { If a patient opts to receive email communication, } \\
\text { program check-ins can be sent via email, allowing } \\
\text { patients to respond when convenient instead of } \\
\text { requiring working-hours availability. }\end{array}$ \\
\hline EHR integration & $\begin{array}{l}\text { Integration with our EHR for } \\
\text { laboratory results, vitals, referrals, } \\
\text { and medications. }\end{array}$ & $\begin{array}{l}\text { Connect to our EHR's laboratory services for relevant } \\
\text { data like basic metabolic panels. }\end{array}$ \\
\hline Device integration & $\begin{array}{l}\text { Ingestion and analysis of home, } \\
\text { cellularly-enabled blood pressure } \\
\text { blood pressures. }\end{array}$ & $\begin{array}{l}\text { Cellularly-enabled blood pressure cuffs can transmit } \\
\text { automatically into the CRM system. }\end{array}$ \\
\hline
\end{tabular}

Abbreviations: CRM, customer relationship management; EHR, electronic health record. 

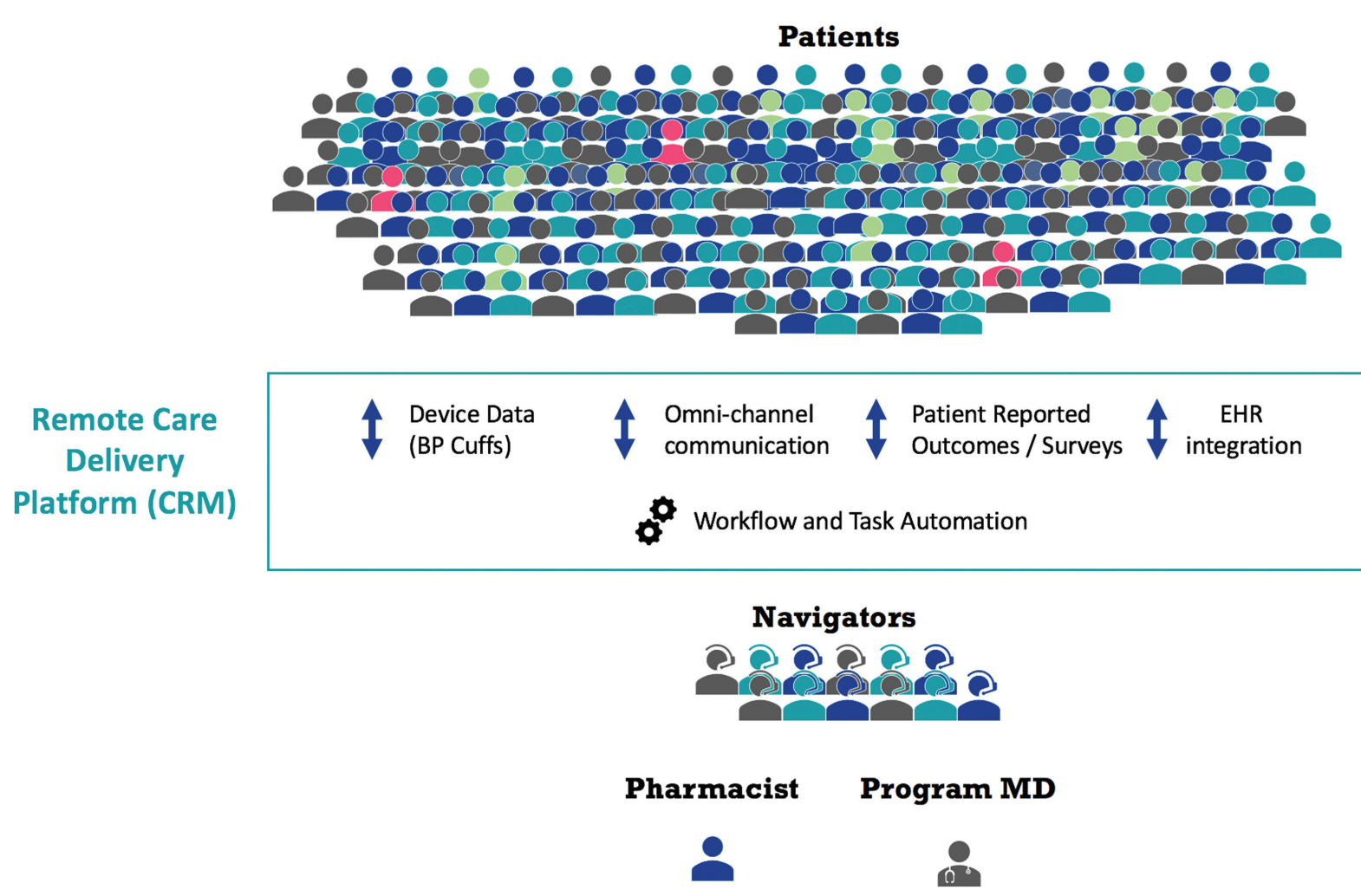

Fig. 1 CRM platform. Our CRM platform enables navigators, physicians, and pharmacists to leverage key pieces of functionality-device data integration, omni-channel communication, patient-reported outcomes and surveys, and EHR integration, powered by workflow and task automation, to manage substantially more patients than could be managed without these technical capabilities. Clinical navigators, using the tools in the box, can deliver guideline-driven, remote care. Because of this model, many more patients can be managed than would be possible with a more traditional 1:1 provider:patient relationship. CRM, customer relationship management; EHR, electronic health record.

blood pressure cuff device data are also integrated and flows directly from cellularly-enabled devices into the system. - Fig. 1 describes how these features come together so that a tiered team consisting of a navigator, pharmacist, and physician, are able to manage more patients than would be possible without the system. The main users of the CRM system are clinical navigators and pharmacists. The system does require training, but most users have been able to start using it to complete tasks the first time they log into the system, with more independence coming over several weeks of regular usage.

\section{Clinical Program and Workflow Automation}

Patients can be referred to our program by primary care or specialty providers through a referral order in our EHR. Patients are guided through a series of stages before transitioning to a "maintenance" phase once clinical targets have been achieved and where they then receive follow-up at longer intervals. Below, we highlight the five main phases, along with the key places where workflow processes have been automated. - Table 2 provides specific examples of process improvements and workflow automations within each of the five stages.

\section{Intake and Eligibility Screening}

In this phase, patients are brought into the system automatically through EHR data extracts or referrals. There is an initial screening process to look for exclusion criteria (for example, age and weight restrictions due to blood pressure cuff size, or clinical contraindications such as dialysis or significant heart failure). Patients are then sent a survey, either through our portal or via email, to confirm interest in the program, specify communication preferences (phone, email, portal, or SMS), and consent for communication per organizational policy (e.g., for SMS or email). Once communication preferences are established, program communication preferentially takes place through that medium. Reminders to complete the survey, as well as tasks to call patients if they do not complete the survey electronically, are created by the CRM system automatically and assigned to navigators.

\section{Enrollment}

Patients who complete the initial survey are then contacted via the communication preference of their choice to confirm their mailing address, and then mailed a cellular blood pressure cuff with instructions on setup and usage, for example, best practices for taking a blood pressure, as well as timing (two readings in the morning and night, while sitting). At this point, patients are considered enrolled in the program.

\section{Device Configuration}

The blood pressure data are integrated into the CRM system, so that once a patient starts checking their blood pressure, it 
Table 2 Workflow automation and process improvement

\begin{tabular}{|c|c|}
\hline Stage & Process improvements and workflow automations \\
\hline $\begin{array}{l}\text { Intake and eligibility } \\
\text { screening }\end{array}$ & $\begin{array}{l}\text { - Referrals automatically ingested by CRM system, so that patients are not loaded manually. } \\
\text { - Eligible patients are asked to self-enroll through an automatically generated survey, minimizing } \\
\text { phone calls (though still enabling patients to be called if they do not engage electronically). } \\
\text { - Reminders to follow-up with patients at specified time periods are created by the CRM system and } \\
\text { assigned to navigators. }\end{array}$ \\
\hline Enrollment & $\begin{array}{l}\text { - CRM system uses "just-in-time" process to collect data only when needed, for example, confirming } \\
\text { the patient's address only when they've agreed to participate in the program. } \\
\text { - Confirmation of mailing address triggers a process by which device procurement starts. }\end{array}$ \\
\hline $\begin{array}{l}\text { Device configuration } \\
\text { and setup }\end{array}$ & $\begin{array}{l}\text { - Blood pressure data automatically flows into CRM system, linked to patient during procurement } \\
\text { process. } \\
\text { - Tasks are created to automatically remind patients to check their blood pressure (with instructions) if } \\
\text { blood pressure data are not coming into the system. } \\
\text { - Once a patient has sufficient blood pressure data to make a clinical decision, the system moves the } \\
\text { patient to the next stage in the overall program workflow. }\end{array}$ \\
\hline Medication titration & $\begin{array}{l}\text { - Navigator puts together clinical summary of patient only when that patient is ready to be titrated, } \\
\text { e.g., has sufficient blood pressure readings. } \\
\text { - Navigator and pharmacist use task-based workflows to review patient and guide clinical management } \\
\text { decision and communication. }\end{array}$ \\
\hline Maintenance & $\begin{array}{l}\text { - The CRM system creates future reminders (e.g., } 6 \text { months) that are automatically sent to the patient } \\
\text { to encourage resuming blood pressure checks. } \\
\text { - The clinical management workflow resumes once the patient's device data starts flowing again; if it } \\
\text { does not, tasks are created to attempt other methods of reaching patient based on their } \\
\text { communication preferences. }\end{array}$ \\
\hline
\end{tabular}

Abbreviation: CRM, customer relationship management.

is automatically linked to their record using a device ID mapped to the patient during device procurement. Since the blood pressure cuffs are cellularly enabled, no further setup is required on the patient's behalf (if a patient is unable to use the cellular device, or there is a cellular transmission problem with their geographic location, they are sent a noncellular cuff and the system reverts to manual processes to collect the data). The system waits 7 days to determine if blood pressure data starts flowing into the system, and if none is captured, the system creates tasks to follow-up with the patient to ensure proper setup and training if needed. Once a patient has submitted enough blood pressure readings to form a clinically appropriate "average" (typically three or more days of blood pressures checked twice in the morning, twice at night), the patient is moved to the titration step.

\section{Medication Titration}

Once a patient's blood pressure has been averaged, the patient's clinical summary is put together by a navigator and sent to a clinical pharmacist for review through the CRM system. Data gathered by the navigator includes past medical history, relevant laboratory data, current and prior medications, medication intolerances and allergies, and the patient's blood pressure data, supported by integration with the EHR. The clinical pharmacist, supported by an algorithm that takes similar data as inputs, will then make treatment decisions, and the pharmacist will prescribe a medication and/or order a laboratory test. If no medication adjustment is needed (for example, the patient's blood pressure is under their guideline-driven goal, 130/80 for most patients), then the navigator will move the patient into the maintenance stage. If the patient's blood pressure is not at goal, then the patient will start the medication titration phase over again once they have adjusted their medications.

\section{Maintenance}

Once a patient's blood pressure is at goal, they enter the maintenance stage. They are encouraged to continue to take their medications as prescribed, provided educational material around lifestyle modifications, and the system creates a task to contact the patient in 6 months to repeat their blood pressure check. At 6 months, processes such as automated SMS or email reminders, using patient preferences, automatically urge the patient to resume blood pressure monitoring.

Of note, not all patients will flow through this exact staged approach. For example, patients who report lightheadedness are automatically routed to a workflow to be evaluated for orthostatic hypotension prior to entering the titration phase. Some patients opt for lifestyle modifications instead of medication management, and there is a pathway for patients who require higher-touch clinical care, for example, a patient who has resistant hypertension or numerous comorbidities and medication intolerances. Additional components of our program include daily monitoring for blood pressures that are "out of bounds" (too high or too low), which are then routed to clinicians for manual review outside of the CRM system to ensure patient safety for critical results. These results are reviewed by dedicated program clinical staff daily 


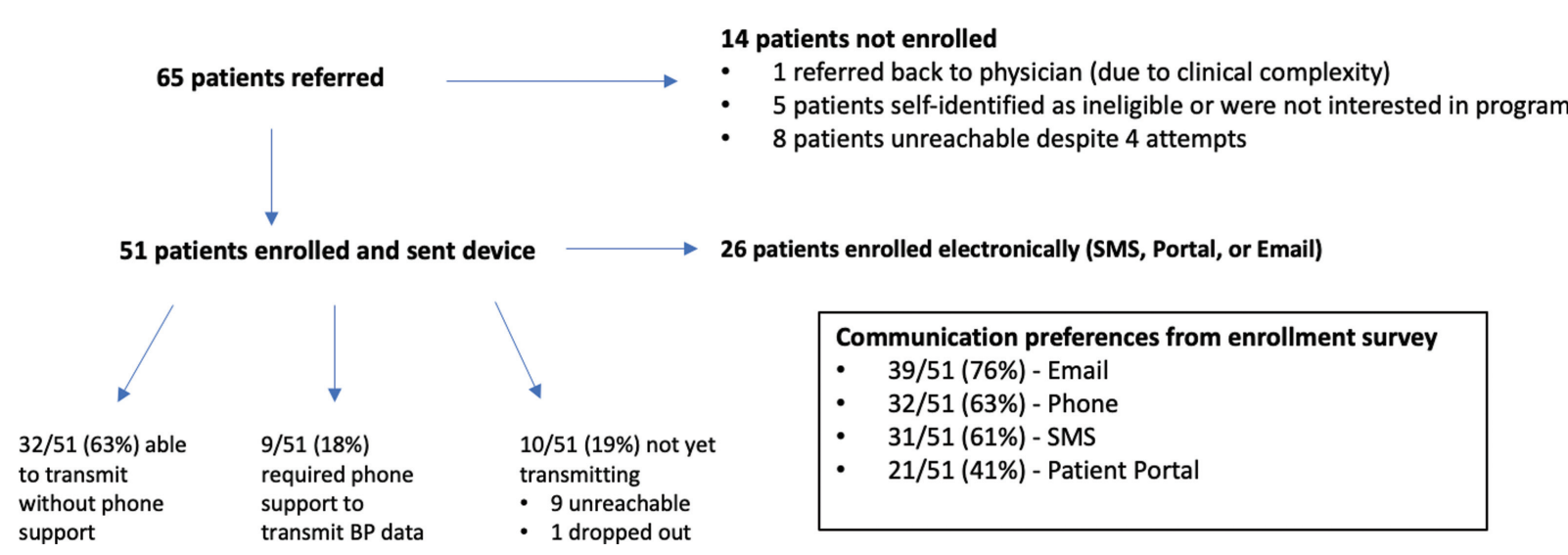

Fig. 2 Initial enrollment metrics. Many of the patients referred to our program were able to enroll and get their personal device configured and transmitting using electronic communication exclusively, highlighting the places where workflow automation could improve processes.

and are not sent back to referring providers unless clinically appropriate.

\section{Integration with the Electronic Health Record System} Our CRM system is designed to augment, but not replace, our EHR, and our navigators use the EHR and our CRM system concurrently. We have implemented several unidirectional integrations. For example, the EHR is the source of truth for patient identity, and we use a web service tied to our master patient indexing system to ensure we have the most current patient demographics, which are still managed centrally (except for program-specific variables, like communication preferences). We also receive inbound referrals and laboratory values through EHR integrations. Our navigators use on the EHR for patient chart review, documenting encounters, and communication where appropriate (like messaging other providers through the native EHR messaging system).

\section{Initial Results}

Of the first 65 patients referred to our program, 51 were ultimately enrolled (e.g., confirmed interest in participating and sent a blood pressure cuff), with more than half enrolled using email, SMS, or our patient portal, and not requiring a phone call (-Fig. 2). Most of our patients indicated email as their preferred communication method in the enrollment survey $(39 / 51,76 \%)$, while the patient portal was the least desired $(21 / 51,41 \%)$. Many patients were able to transmit their BP data without requiring phone support-32/51 (63\%), while only $9 / 51$ (18\%) required phone support, with the remainder either not yet transmitting, dropped out, or ultimately unreachable.

\section{Discussion}

We describe the design and features of a CRM system, built to support a navigator and pharmacist-led virtual care program for hypertension management. This system has numerous features that result in improved clinical processes and enhanced workflows, like task automation, multi-channel communication capabilities, and integrations with devices and our EHR. Additionally, the CRM system enables workflow automation across all five stages of the program, from intake and enrollment through longer-term maintenance. The system allows our team to focus on clinical care, patient communication, and patient education as opposed to manual task tracking. Our initial results suggest patients prefer electronic communication, and that many processes, such as blood pressure device setup and transmission, are amenable to automation.

The role of information technology in creating more productive, efficient, and streamlined processes is debated within health care and other industries. Researchers have articulated a "productivity paradox" that describes disappointing improvements-or even sometimes worsening-of productivity as information technology is introduced to various industries. ${ }^{17}$ This is particularly salient for health care given large investments in health care information technology internationally, particularly in the United Sates, over the past decade. ${ }^{18}$ Workflow-the set of steps required to complete an activity-drives much of what our health care workforce does across the entire lifecycle of care delivery, from front-line clinicians to back-office infrastructure and staff. Building systems that are specifically targeted at improving workflow could improve patient safety, efficiency, as well as satisfaction of patients and providers. ${ }^{19-21}$

CRM software is not new to health care. Occasionally called "patient relationship management" to emphasize the patient-as-the-customer, many organizations have begun to focus on the "patient" relationship, including implementation of CRM software or hiring patient experience executives. $^{15,22,23}$ There are dozens of health care-based CRM offerings, with an estimated market size of $\$ 8.8$ billion. ${ }^{24}$ CRM can serve many functions at a health care organization -for example, managing patient volunteers, billing collections, or fundraising. We have built our CRM system to enable direct care delivery, using the automation and task management to allow navigators and clinical pharmacists to manage patients under the supervision of expert physicians. To our knowledge, our CRM system is the first to focus on hypertension management.

Ensuring equitable access for as many patients as possible is a core priority of our clinical program, and is evident in our overall program strategy-as a virtual program, we broaden overall access by not requiring in-person visits. We also use 
cellular blood-pressure cuffs, so that home broadband is not required, and no smartphone or tablet devices are necessary for device integration. Our CRM implementation further strengthens our ability to provide equitable access to our clinical program. Because we have multi-channel communication capabilities, we can interact with patients in several ways, based on patient preference. For example, portal messaging, email, and SMS allow patients to respond at their convenience (instead of only having phone communication which is typically done during working hours). But because we are tracking patient preferences, we can also call them-if a patient does not respond to an electronic communication, we can always fall back on a phone call. Our CRM platform also natively supports multilingual processes, expanding our patient reach. Further ways of expanding access to our program-like through community health workers-is an area we are exploring.

There are notable limitations to our implementation as well as CRM in general. First, there is typically less flexibility with a CRM platform than a custom application. CRM software comes with "out of the box" functionality that can greatly accelerate development, but also limit flexibility. For example, user interface design is often proscribed by the CRM software, and certain buttons or headers cannot be removed, or can only be minimally modified, though the advantage is a consistent user experience across applications. Second, most CRM solutions are cloud-based offerings, and as a result, releases and updates are pushed down to customers, creating higher risk for "breaking" changes. It is important to have mitigations in place should a release negatively impact application behavior. Of course, there is also an advantage to this model-we can leave some aspects of security, hosting, and many application operational processes to our CRM provider, and critical patches are available immediately.

Finally, our CRM implementation is early; we launched in November of 2020 and have been building out the platform iteratively. While we present some initial results here, further work is needed to understand where these potential improvements in efficiency will materialize, specifically focused on process metrics and clinical outcomes. For example, we are interested in clinical outcomes, like sustained systolic blood pressure reduction. However, since we have already shown these improvements in our existing program, ${ }^{7}$ we will evaluate whether we can reach a broader set of patients now that we are using electronic communication. Additionally, we will evaluate program efficiencylike how long navigators spend per task, per patient, over time, and whether there are continued opportunities to improve operational program performance.

\section{Conclusion}

In conclusion, we have implemented a CRM solution that supports a navigator and pharmacist-led, algorithmically supported, virtual hypertension management program. Our implementation has introduced numerous improvements in operational processes including enhanced workflow tools and automation. Future work will examine the operational improvements of this health information technology solution.

\section{Clinical Relevance Statement}

Customer relationship management (CRM) systems can be used to improve clinical processes, enabling more efficient management of patients through features like multi-channel communication, workflow automation, and device integration. CRM systems, like the example described here, can enable clinical programs to scale to more patients and could provide value for numerous use cases.

\section{Multiple Choice Questions}

1. Which of the following is an important capability of customer relationship management (CRM) systems for improving clinical processes describe here?

a. Task automation.

b. Heart rate measurement.

c. Wireless transmission of pacemaker readings.

d. Medication dispensing capabilities.

Correct Answer: The correct answer is option a, task automation. While CRM systems have numerous capabilities, the ability to improve processes through task automation is a critical example of how this implementation will improve overall clinical workflow and processes. For example, automatic reminders to follow-up on key clinical tasks.

2. When implementing a virtual hypertension management program, which of the following is an example of workflow automation that could improve program efficiency?

a. Patients are required to fill out a program intake survey.

b. Referring providers must call our office before a patient is officially enrolled.

c. Patients receive a system-generated SMS instead of a phone call if they need to transmit more blood pressure data.

d. Navigators call local pharmacies to confirm a patient's medication list.

Correct Answer: The correct answer is option c, patients receive a system-generated SMS instead of a phone call if they need to transmit more blood pressure data. Improving how patients interact with our clinical program is an important component of CRM, and automating follow up-for example, sending an SMS reminder to a patient instead of requiring a manual phone call-is an example of workflow automation that could improve program efficiency.

Protection of Human and Animal Subjects

This work is a quality improvement project at Mass General Brigham whose sole purpose is quality measurement and thus under institution guidelines the work was 
not reviewed by the Mass General Brigham Human Research Committee.

\section{Conflict of Interest}

W.J.G. reports consulting income from the Office of the National Coordinator for Health IT and Novocardia, Inc., both outside the scope of this work. B.M.S. reports institutional research grants to Brigham and Women's Hospital from AstraZeneca, Eisai, Merck, Novartis, NovoNordisk, and Pfizer and consulting fees from Allergan, Boehringer Ingelheim, Elsevier Practice Update Cardiology, Esperion, Hamni, Lexicon, Medtronic, and NovoNordisk.

\section{References}

1 Yoon SS, Gu Q, Nwankwo T, Wright JD, Hong Y, Burt V. Trends in blood pressure among adults with hypertension: United States, 2003 to 2012. Hypertension 2015;65(01):54-61

2 Blood Pressure Lowering Treatment Trialists' Collaboration. Pharmacological blood pressure lowering for primary and secondary prevention of cardiovascular disease across different levels of blood pressure: an individual participant-level data meta-analysis. Lancet 2021;397(10285):1625-1636

3 Jones DW, Whelton PK, Allen N, et al. Management of stage 1 hypertension in adults with a low 10 -year risk for cardiovascular disease: filling a guidance gap: a scientific statement from the American Heart Association. Hypertension 2021;77:e58-e67

4 Muntner P, Hardy ST, Fine LJ, et al. Trends in blood pressure control among US adults with hypertension, 1999-2000 to 20172018. JAMA 2020;324(12):1190-1200

5 Rethy L, Shah NS, Paparello JJ, Lloyd-Jones DM, Khan SS. Trends in hypertension-related cardiovascular mortality in the United States, 2000 to 2018. Hypertension 2020;76(03):e23-e25

6 World Health Organization. Hypertension. Accessed April 16, 2021 at: https://www.who.int/westernpacific/health-topics/ hypertension

7 Scirica BM, Cannon CP, Fisher NDL, et al. Digital care transformation: interim report from the first 5000 patients enrolled in a remote algorithm-based cardiovascular risk management program to improve lipid and hypertension control. Circulation 2021;143(05):507-509

8 Weaver KK. Collaborative practice agreements: explaining the basics. Pharm Today 2018;24(03):55

9 Wright A, Salazar A, Mirica M, Volk LA, Schiff GD. The invisible epidemic: neglected chronic disease management during COVID19. J Gen Intern Med 2020;35(09):2816-2817

10 Fisher NDL, Fera LE, Dunning JR, et al. Development of an entirely remote, non-physician led hypertension management program. Clin Cardiol 2019;42(02):285-291

11 Blood AJ, Fischer CM, Fera LE, et al. Rationale and design of a navigator-driven remote optimization of guideline-directed med- ical therapy in patients with heart failure with reduced ejection fraction. Clin Cardiol 2020;43(01):4-13

12 Chen Injazz J. Popovich Karen. Understanding customer relationship management (CRM): People, process and technology. Bus Process Manag J 2003;9(05):672-688

13 Gartner. Gartner Says Worldwide Customer Experience and Relationship Management Software Market Grew 15.6\% in 2018. Published June 17, 2019. Accessed April 19, 2021 at: https:// www.gartner.com/en/newsroom/press-releases/2019-06-17-gartner-says-worldwide-customer-experience-and-relati

14 HIMSS Interoperability \& Health Information Exchange Community. HIE Case Study: CRM Solutions in Elder Care-The Maplewood.; 2019. Accessed July 12, 2021 at: https://www.himss.org/sites/hde/ files/media/file/2020/07/21/himss_crm_casestudy_themaplewood.pdf

15 Baashar Y, Alhussian H, Patel A, et al. Customer relationship management systems (CRMS) in the healthcare environment: a systematic literature review. Comput Stand Interfaces 2020; 71:103442-103442

16 Sanchis R, García-Perales O, Fraile F, Poler R. Low-code as enabler of digital transformation in manufacturing industry. Appl Sci (Basel) 2019;10:12

17 Jones SS, Heaton PS, Rudin RS, Schneider EC. Unraveling the IT productivity paradox-lessons for health care. N Engl J Med 2012; 366(24):2243-2245

18 Halamka JD, Tripathi M. The HITECH Era in retrospect. N Engl J Med 2017;377(10):907-909

19 Unertl KM, Novak LL, Johnson KB, Lorenzi NM. Traversing the many paths of workflow research: developing a conceptual framework of workflow terminology through a systematic literature review. J Am Med Inform Assoc 2010;17(03): 265-273

20 Zheng K, Ratwani RM, Adler-Milstein J. Studying workflow and workarounds in electronic health record-supported work to improve health system performance. Ann Intern Med 2020;172 (suppl 11):S116-S122

21 Carrera A, Pifarré M, Vilaplana J, et al. BPcontrol. A mobile app to monitor hypertensive patients. Appl Clin Inform 2016;7(04): 1120-1134

22 Poku MK, Behkami NA, Bates DW. Patient relationship management: what the U.S. healthcare system can learn from other industries. J Gen Intern Med 2017;32(01):101-104

23 Yaghoubi M, Asgari H, Javadi M. The impact of the customer relationship management on organizational productivity, customer trust and satisfaction by using the structural equation model: a study in the Iranian hospitals. J Educ Health Promot 2017;6:6

24 PRNewswire. Global Healthcare CRM Market 2018-2023 Featuring Key Players. Published November 22, 2018. Accessed April 20, 2021 at: https://www.prnewswire.com/news-releases/globalhealthcare-crm-market-2018-2023-featuring-key-playerssalesforce-sap-oracle-microsoft-ibm-influence-health-sugarcrm-accenture-healthgrades-and-infor-300754682.html 